\title{
INTEGRATION OF BLOCKCHAIN TECHNOLOGIE IN CASE OF SYSTEMS ENGINEERING AND SOFTWARE ENGINEERING IN AN INDUSTRIAL CONTEXT
}

\author{
Salehi, Vahid \\ Munich University of Applied Sciences
}

\begin{abstract}
Currently, inconsistent software versions lead to massive challenges for many car manufacturers. This is partly because within the product lifecycle management and the software engineering process, there is no correct handling of software versions for the "data entry" (installation of software on the ECU) of the vehicles. Furthermore, there are currently major challenges for many vehicle manufacturers to ensure transparency, integrity and full traceability of SW data status vis-à-vis the legislator. To counteract these challenges, new solutions in the field of vehicle engineering are to be developed based on a new platform called "CarEngChainNet" and Blockchain technology. On the basis of the "CarEngChainNet" platform, new main and sub-chain chains will be developed that allow tamperproof SW data management (Peer to Peer and crypto technology) across the entire PLM chain with new methods such as model-based systems engineering of the requirement, function and integration of the SW components in different areas of vehicle development. The aim is to develop new transmission chains of vehicles with individually packaged software artefacts (e.g. ECU software) that can be securely transmitted from server to server into the vehicle.
\end{abstract}

Keywords: Systems Engineering (SE), Product Lifecycle Management (PLM), Design methods

Contact:

Salehi, Vahid

Munich University of Applied Sciences

Mechatronics

Germany

salehi-d@hm.edu 


\section{INTRODUCTION}

In addition to the hardware, the software is an elementary component of a vehicle. Each control unit has a software with associated data sets and parameters for the full guarantee of the functions. The software and data sets are derived from the functional description developed by the engineering department. All part numbers of the parameters, the software and the data records within a control unit are stored in a vehicle data file. A vehicle data file is a text file that can be opened in an editor and contains all relevant information about the vehicle such as identification number Vehicle Identification Number (VIN), version number, previous version number and colour of the vehicle.

These VINs are quite distinct and they help to identify the vehicles. The related paper certificates and even the locations where the VIN is stored inside a vehicle are far from being tamper-proof. Another problem is that these VINs also fail to generate a proper history of a vehicle based on various databases. That is why according to the Mobility Open Blockchain Initiative (MOBI) press release (2019), Blockchain based VINs will be helpful in creating the digitization of many mobility use-cases such as maintenance history, usage-based insurance, micro-transactions etc., and in short an existence of a vehicle's digital twin, as explained by the MOBI Working group's first draft version on VIN, seen in the Figure 1.

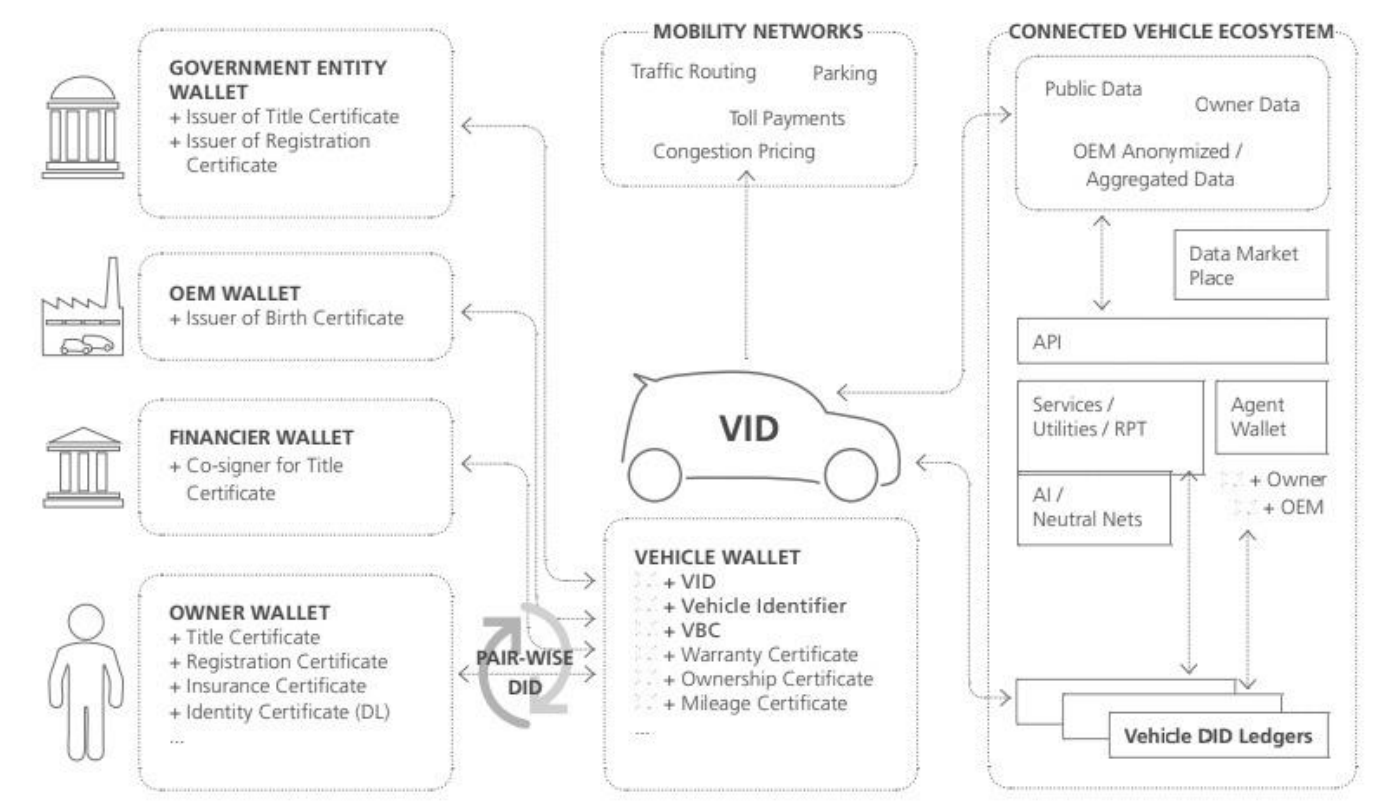

Figure 1. Diagram of the draft version of a first Blockchain-based Vehicle Identity Standard

(Source and (): MOBI / VID Working Group)

In the research project "CarEngChainNET", the possibilities of the latest industry 4.0 and digitization technologies, such as the use of Blockchain (BC) in the field of Model-Based Systems Engineering (MBSE) with the associated Product Data Management (PDM) and Product Lifecycle Management (PLM) information, are considered holistically. A Blockchain platform called "CarEngChainNet" (Proof of Concept) is to be researched and developed in the area of engineering for the management of software data (data input for control units on the basis of legislation) and engineering data within product lifecycle management. At this point, data input refers, for example, to the uploading of software versions to a control unit in the vehicle. Figure 2 shows the two most important directions of the research project, which shows the use of Blockchain technology (with the platform CarEngChainNet) in connection with engineering Data aspects. It is possible to present and view all product-relevant requirements, functions and logical aspects of the product up to the usage phase in a holistic and consistent way. The CarEngChainNet platform will be used to research and apply new Blockchain technologies at a Truck \& Bus Original Equipment Manufacturer (OEM). 


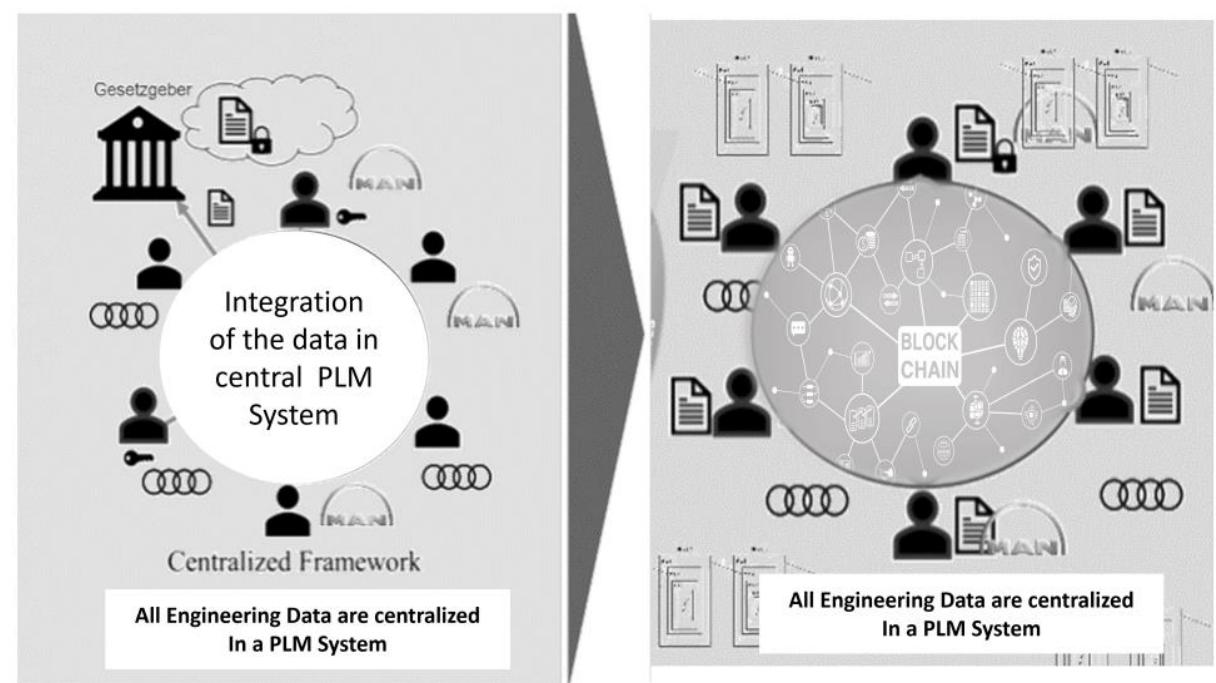

Figure 2. Using Blockchain for Engineering Data decentred instead of centralized PLM Systems

The selected Truck company provide their environment for the development of these innovative ideas and develop new processes, data and systems for the integration of the Blockchain platform. For this development, new methods such as MBSE in conjunction with PLM, are to be researched and developed. The selected Truck OEM also has many years of expertise in the field of Blockchain technology and will integrate system integration based on e.g. "Blockchain Ethereum" or an open source "chain-net" solution. With the newly developed generic Blockchain approaches, such future "game changer" technologies are to be examined for robustness in an industrial environment. Using the CarEngChainNet platform, functions such as software engineering for autonomous driving, functional safety, more transparency, data integrity, consistency and counterfeit protection are achieved based on various application cases. The potentials of Blockchain, as discussed by Schollmeier (2001), in the area of product data and lifecycle management, which also includes software protection and data entry for autonomous vehicles, are to be researched and examined from the point of view of processes, data, architectures, systems and methods. As a result, a Blockchain network called "CarEngChainNet", which is necessary for all participants, is to be developed for these special applications. On the basis of the results, it will then be investigated to what extent a decentralised and networked system based on Blockchain and crypto technology (hash technology) with peer-to-peer networking and Merkle structures is advantageous over more centralised data management systems currently used by many companies. Simply defined Merkle tree root is the hash of all the hashes of all the transactions that are part of a block in a blockchain network. In cryptography and computer science, a hash tree or Merkle tree is a tree in which every leaf node is labelled with the cryptographic hash of a data block, and every non-leaf node is labelled with the cryptographic hash of the labels of its child nodes. Hash trees allow efficient and secure verification of the contents of large data structures. Hash trees are a generalization of hash lists and hash chains. Hashing as defined in literature as the process of producing a fixed-size output from given input of variable size. This is achieved through the use of sophisticated hash functions (implemented as hashing algorithms). Common hashing functions among others like the famous Secure Hash Algorithm (SHA-256) algorithm produces an outputs of 256 bits.

The aim is to explore how this approach can be technologically designed and implemented. It should be questioned whether a new quality of consistency, transparency, process, data and method integrity and thus an acceleration of the automation of processes (requirements, functions, logical view) can be achieved, as illustrated in Salehi et al. (2009a, 2009b, 2009c). The goals are discussed in the following subsections.

\section{Goal 1: Blockchain in Engineering}

Identities of those involved in the engineering process should be able to be determined more transparently in the future in order to track engineering-relevant transactions and at the same time make them secure and not visible to everyone. The extent to which this can be achieved on the basis of 
available blockchain technology approaches, i.e. within a decentralized network based on cryptography and digital signatures, and how advantageous this is compared to other "conventional" approaches, will be evaluated.

\section{Goal 2: Legal obligations to provide evidence}

By using a decentralized Blockchain network, more traceability, consistency, transparency, integrity of the Functional Safety, $\mathrm{CO} 2$ and emission product data can be achieved both on the software side and on the hardware side with regard to the requirements of the engineering processes based on Systems Engineering. The aim is to investigate the extent to which Blockchain can provide a basis for novel, value-adding processes, methods and tools that meet the above requirements better than conventional (in the sense of non-Blockchain-based) implementation alternatives.

\section{Goal 3: Bottlenecks \& Single Point of Failures}

The aim is to investigate the extent to which the realization of a decentralized system accelerates engineering processes.

\section{Goal 4: Democratization of Engineering Processes \& Change Management}

To enable the integrity of data to be validated by many interdisciplinary Systems Engineering staff in the network, as per the approaches discussed and elaborated in Salehi et al. (2009c) and Salehi et al. (2011a, 2011b) and to reach a common consensus on which data to add to the chain. It will be examined whether a democratisation of the engineering processes in the company is achieved and whether it is possible to break up "silos" in the company since the Blockchain. To be able to handle the change management of the engineering Data every data format will be hashed and marked with an hash number. Furthermore after the first generation of the genesis number for each generated later version of the engineering files will be marked up with an hash block.

\section{Goal 5: Maximum safety of engineering data}

The engineering specific mechanisms that make it as difficult as possible for external attackers to manipulate existing engineering data are to be identified.

\section{Goal 6: Integrate engineering data}

This aspect includes the integration of the engineering data which includes for example requirement management, functional engineering and CAD design. Therefore by means of Blockchain the correct data will be hashed and integrated in the blockchain network. It is examined which processes upstream of the Blockchain must be adapted or automated accordingly.

\section{STATE OF THE ART IN SCIENCE AND TECHNOLOGY}

Since Blockchain technology is only at the beginning of its development, no uniform definitions have yet been adopted, according to Mattila (2016) and Swan (2015). Schollmeier (2001), Schoder et al. (2002) defines a Blockchain as an electronic register for digital records, events or transactions managed by subscribers of a distributed computer network. This definition makes it clear that a distinction must be made between the Blockchain as a data structure and the associated management system. Walport (2015) defines a Blockchain as a kind of database in which entries are grouped into blocks. These blocks are linked in chronological order by a cryptographic signature, as described in the works of Bogart et al. (2015), Walport (2015). Each block contains records of valid network activity since the last block was added, as mentioned in Bogart et al. (2015). In the case of Bitcoin the world's largest cryptocurrency, for example, this includes the transactions that are being carried out. Walport (2015) distinguishes Blockchains from distributed ledgers in which recordings are stored continuously instead of in blocks. In this study, there is no differentiation between the terms Blockchain and distributed ledger due to the application-oriented focus. Glaser et al. (2015) describes the associated management systems as distributed consensus systems. According to the authors, such systems are based on cryptography and peer-to-peer (P2P) principles rather than a central authority to achieve network-wide verification of the system's status by consensus, as discussed in Glaser et al. (2015). Such P2P architecture of a blockchain allow transactions to be transferred across geographical boundaries, eliminating and bypassing the middle men. In this study, comparable systems are referred 
to as Blockchain systems. The above definitions show that Blockchain systems are distributed systems. According to the work of Mullender (1990) the distributed systems cannot be exactly defined, but rather characterized by several properties. First, they have several independent computers (network nodes) that communicate and synchronize with each other. The failure of individual computers does not affect other computers. In addition, each network node stores a common status of the system, so that the failure of individual computers does not imply the (partial) loss of the system status. In Blockchain systems, the data of the Blockchain is stored in each node (redundantly).

The peer-to-peer principles mentioned by Mullender (1990) also include the following properties. The network participants provide hardware resources to provide contents or services of the network according to Schollmeier (2001) and Salehi et al. (2018). In addition, there is a direct exchange between the nodes, i.e. there is no central instance to coordinate the communication between the individual network nodes, according to Schoder et al. (2002). Furthermore, the above definitions show that Blockchain systems use cryptography, as well as the consensus mechanism also mentioned, by means of which the network nodes coordinate the system status, and which can be regarded as the fundamental innovation behind Blockchain systems. A consensus mechanism ensures the authentication without the need for central authority. All the participants in such consensus mechanism, have to follow and apply the defined rules and accordingly a transaction is agreed and accepted.

There is a growing complexity in the architecture and the wide variety of software applications running on the Electronic Control Unit (ECU). It is necessary for OEMs to handle the software during the vehicle's entire life cycle efficiently, besides ensuring the security. Many OEMs today rely on Over-The-Air (OTA) technologies. The main objective behind using such OTA updates is to offer a great amount of help in maintenance to prevent the frequent recalls or the repetitive repair shop visits that makes the customer experience not so positive. Besides updates and bug fixes, OTA also allows users to upgrade autonomous car functions like any updates in adaptive cruise controller, lane-keeping assistant or just an extra power on demand. Leveraging on blockchain technology can help in generating trust, thanks to immutable nature of the blockchain and at the same time greatly reduce the susceptibility of significant security threats to OEMs when performing such OTA updates. Riddle\& Code (2019) whitepaper cites that, with the rapid developments in field of autonomous vehicles, such cybersecurity challenges will continually grow and the existing bus systems such as the CAN bus or On Board Diagnostic (OBD) protocols are not quite secure enough and technically outdated, but at the same time are quite important to the OEMs. Hence, there is a dire need for securely carrying out OTA updates.

According to Heber et al. (2017), normally in the case of ECUs, there are a variety of components or artefacts. Typically they would be composed of at least one hardware. The different hardware components such as CPU, plugs, pins etc. together make one big association in a hardware. Such hardware components are generally said to be less volatile with regard to their persistence because of the fact that ECU is replaced only in garage service, if at all a defect is detected. So it is clear fact that, to provide high flexibility within a documentation system, does not come mainly from hardware changes. Additionally, several parts of software also are information artefacts within the specifically defined combination. Software is subject to many changes due to a very high complexity in its code structure and hence highly prone to errors. Additionally, today customer demands have grown and their expectations include connecting their own smartphone to car infrastructure over the internet, personal applications etc. According to Heber et al. (2017) these functions and interfaces are more related and integrated in the car and outside of it. Such functions are more and more realized and executed by software today. This very high volatility and complexity yields needs traceability.

Immediate software fixes, or even the feature upgrades for ensuring better customer experience make the tasks challenging. Hence, a continuous documentation basis and the traceability becomes important.

According to Heber et al. (2017), this arises to the research question like: How could the traceability of such control units in the lifecycle of a vehicle be improved? It is possible to ensure more traceability across all the discipline according to the new approach - Munich Agile MBSE Concept (MAGIC), developed by Salehi V. et al. (2019). 


\section{PROPOSED APPROACH}

The scientific and technical objectives of the project can be described as follows. By using the CarEngChainNet platform, more traceability, consistency, transparency and integrity of the product data both on the software side and on the hardware side are to be achieved with regard to the requirements of the engineering processes based on MBSE (e.g. requirements of legislation with regard to software and data). New processes, methods and tools that are Blockchain capable will be researched and developed. Figure 3 shows the current world of engineering. Currently, all software data, from preparation to administration, is stored centrally on a PLM system. A continuous integration of the relevant software data from the creation to the integration in the vehicle in the area of engineering is only partially available. Furthermore, new engineering approaches such as MBSE are only partially reflected in existing processes. During the development of the elements for the software, data set and function parameters, the generated data objects are managed and specifically compiled and released for a vehicle Figure 3. Security is provided by controlled access (roles and rights). Vehicle manipulation is checked in modern processes by many OEMs by signing elements and managing keys in secured areas in engineering and software development. This enables "end2end" security in the transmission process and prevents manipulation by third parties. Reporting to the authorities is done manually, the affected data must be considered individually and transferred to the report. Proof (evidence) can only be provided via actual/target comparison, which involves a great deal of effort.

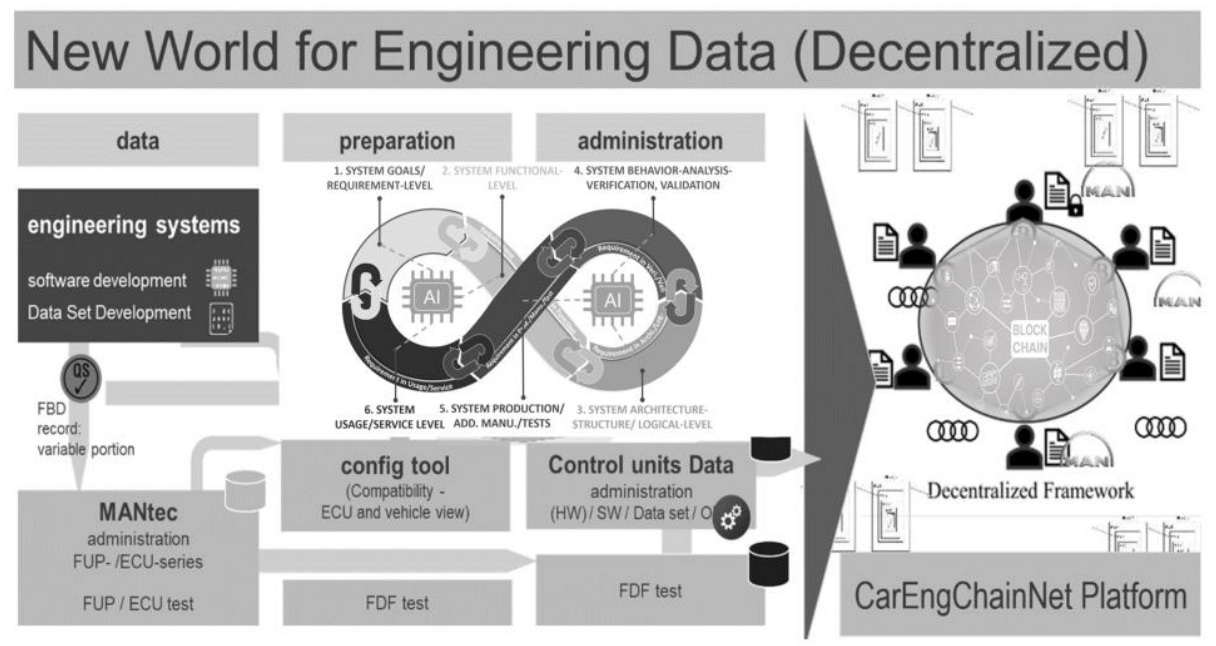

Figure 3. Integration of Blockchain in Engineering

In order to use the Blockchain technology for decentralized software data management for testing, production, service and Over the Air (OTA) functionalities, certain requirements must be met. These requirements include, for example, the clear identifiability of the software components in the generated data set. The provision of metadata and the corresponding geometry data sets to the network are the main focus. The identification and assignment of the software components and assemblies to the blocks is to be maintained for the user, as well as with centralized software data management via software part numbers, version and location code at an internationally established company. The software components in the Blockchain network are identified via the miner structure in the background of the user environment. Therefore, require that on the one hand the data records can be linked to the mining network and on the other hand feedback from the decentralized data structure to the user interface is given. In addition to providing the identification data of the software components, the master data of the software data records must be available to the Blockchain network and its miner structure. The internal encryption of the data set must not be touched by the Miner structure in order to unambiguously assign and couple the software data sets. Changes to internal file encryptions cause problems with common software. Another requirement for the decentralized network is versioning management. The versions shall be characterized by the connection between identification data and block hash through the miner network. This ensures that the individual software versions can be localized in the Blockchain. The possible access of the mining network to all software parts and their 
versions creates the basis for the control unit data. The parameters, alignments and links generated in software development are to be combined and stored in blocks. Therefore, when retrieving the complete software codes, there is a requirement to be able to quickly and safely create the paths and links from the blocks. A necessary condition of the Blockchain is that the last data block carries the hash coding (encryption) of the previous data block and thus forms a continuous data chain. The Blockchain thus places the condition on the network that the participants are always active in the network, that the miner structure can process storage transactions in the network and that the Blockchain is synchronized to the most current state.

\section{STRUCTURE OF THE SOFTWARE BLOCKCHAIN NETWORK}

To set up a decentralized network, it is necessary to set up a suitable infrastructure. This infrastructure must be designed in such a way that the network is always maintained in the event of changes in environmental conditions (e.g. power failure, maintenance, etc.) and that data can be retrieved and stored at any time anywhere in the world. Every software user generates e.g. software data through his development activities. For a large number of developers, this flood of data has to be handled in decentralized data management. In this context, a network of all active participants must be set up in order to be able to retrieve and store the generated data in a decentralized manner via the Blockchain technology. In order to be able to realize this, the network has two separately autonomous data chains. The data records, information and exchange data generated by the software users are to be stored in these two chains. The detailed description of the block information in the two data chains can be found in Section 4. The control function and the management of these two data chains is an important function in the network. With the decentralized software data management, the control function should also be decentralized and cross-network. This control and management function in the decentralized software data management is performed by the Miner. Each active network member has a Miner on its computer. This results in a linked miner network in addition to the computer network. The Miner establishes the computer-computer connection (miner network) as well as the computer-Blockchain connection, as seen in Figure 4. This ensures that every active software user in the network has access to the data chain. This communication model ensures that all participants of the network are synchronized with the data chain and that the status of the data chain is known to the miners.

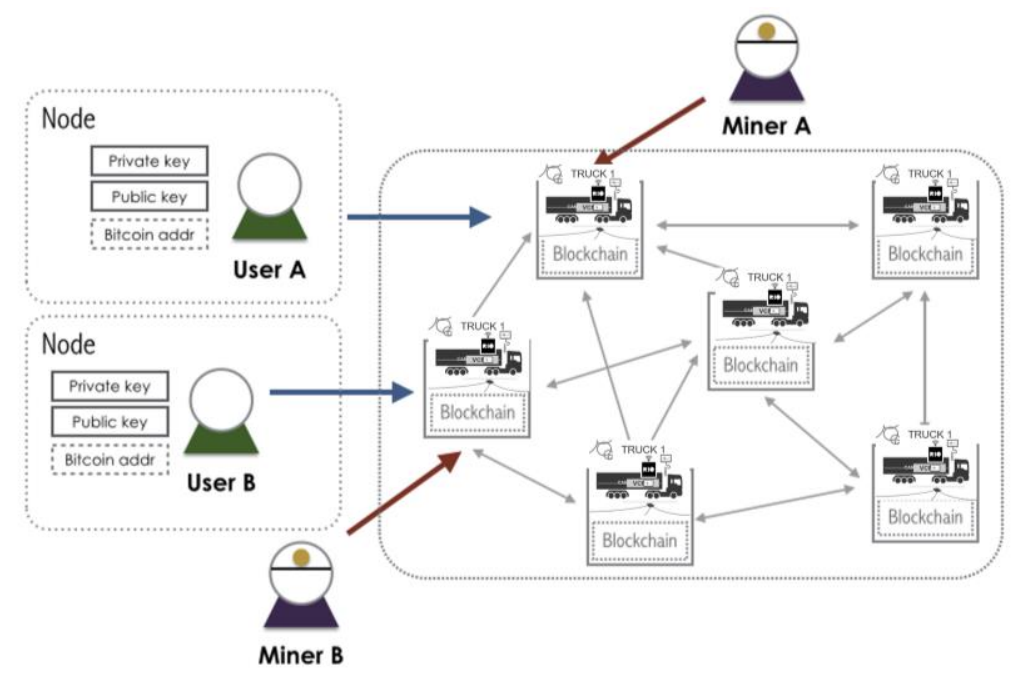

Figure 4. Communication of the Miner Network with the Data Chains

The control function over the legal storage of the data in the chain is carried out by fixed rules in the miner structures. The Miner is also used to check the data to be stored for design rules. When saving a software file of a user in the network, a transaction is triggered by the Miner that wants to save. In this sense, a transaction is understood as the desire to store software data in the data chain. Since there is an active connection between the software users via the miner network, all other miners in the network are notified of this transaction. Apart from the miner of the computer that wants to save, all miners in the network check the conformity. The miners go through the rules anchored in their structure. The storage and design guidelines are checked and compared. To do this, the information contained in the transaction is compared with the storage and design guidelines. The storage guidelines are compared 
in detail with regard to the availability of necessary component information, material data and versions. The comparison is carried out by every miner in the network. If the storage guidelines have been adhered to, the data to be stored is compared with the defined design guidelines in the miner structures. If all information is contained and conforms to the guidelines, all other miners release the transaction to the miner who wants to save. The comparison of the design guidelines with the transaction data via the miner structure ensures that the Blockchain contains only software data that conforms to the company's requirements. The miner of the software user who initiated the transaction request converts the software data into binary code sections and packs these together with the hash coding from the block previously inserted into the chain into a new block. In order to have access to development-specific information, decentralized software data management via blockchain technology must provide an internal interface for this. This not only provides access to development-specific data (e.g. requirements or Advanced driver-assistance systems (ADAS) functions), but also enables software products to be made available via decentralized data management. This interface can be created via the software working group's miner structure. A lead miner communicates with the active main network, which is operated by the software miner network. The background to lead miner communication is that development information is exchanged with the blockchain network via an interface and therefore the synchronization of software component information does not have to be actively carried out between each software user and miner. The software component information from the Blockchain is constantly synchronized via the lead miner in the engineering network so that the system always has the current data available for the individual software components and versions. If a user in the PLM system changes software component-specific information, the lead miner transmits the request to the active network (Software Miner). These localize the block and extract the component-specific data from it. These are transferred via the lead miner to the miner of the Enterprise resource planning (ERP) user and output legibly in the ERP system. The user changes information and requests that the data be saved using a transaction. In the passive network (PLM Miner), the guidelines are checked and then the transaction release is granted. The lead miner then triggers a transaction to the active network (software miner). After the successful check in the active network, a new block in the chain with a higher version is created by the software miner. Due to the property that Blockchain blocks cannot be changed, the block with the higher version differs only in the component-specific changes. The geometry data of the previous version are transferred unchanged to the block with the higher version. The generated hash is also returned by the block as in the active network and communicated to the ERP miners via the software miners and lead miners in the passive network. The structure of the passive network allows access to current PLM-relevant information and synchronized product and component maintenance.

\section{CONNECTION AND CONTENTS OF THE SOFTWARE CHAIN BLOCK}

When software data is stored in the Blockchain and released by the miner network, the software data is stored in the Blockchain by the miner. In order to enable a good performance of the entire Blockchain network and an easier retrieval of the data, the software data is divided and stored in different groups as a block separately in the main or sub chain. These block groups are divided into master data, identification data and links. This means that each user only must load the data records from the Blockchain that he really needs, thus reducing data transfer. Thus, the software user can access all software data, the PLM user can only access the required identification data and the external user can only access the data provided for him. In the main chain, master data and identification data of software components are stored in common blocks. Master data consists of the geometry data, i.e. the main data set of the software file, which is used for the graphic representation in the software programs and the associated links to the respective identification data. Identification data is the information with which an individual part can be precisely identified. These can vary from company to company and must therefore be defined specifically for each company. Identification data can look like the following:

- Software (SW) component number and version,

- $\quad$ local and international names,

- programmers,

- date, 
- functions,

- requirements,

- $\quad$ SW code number,

- $\quad$ current time stamp and

- the corresponding hash.

In the sub chain, on the other hand, link and identification data of the SW component groups and external software data (e.g. vehicle data) are stored, for example, consisting of:

- total SW code,

- name of the codes,

- $\quad$ SW code numbers,

- data from suppliers,

- $\quad$ link to individual SW components,

- $\quad$ current time stamp and

- the corresponding hash.

All master data and identification data must first be extracted from the software file by the Miner when saving the software data and then converted into a binary data format. Only this converted data can be stored in the Blockchain. For example, in the case of individual parts, the metadata mentioned above are read out and stored in the main chain as separate blocks with the current time stamp and the respective hash. The division of the individual parts into the main chain and the assemblies into the sub chain facilitates access to the individual software components. Since assemblies "only" consist of linked individual parts and their orientation in space and their relationships to each other, it makes sense to store assemblies in the sub chain. This facilitates access to software components for software development. Truck OEM or supplier data records are stored exclusively in the sub chain in order to achieve a clear separation of internal and external software components, as seen in Figure 5.
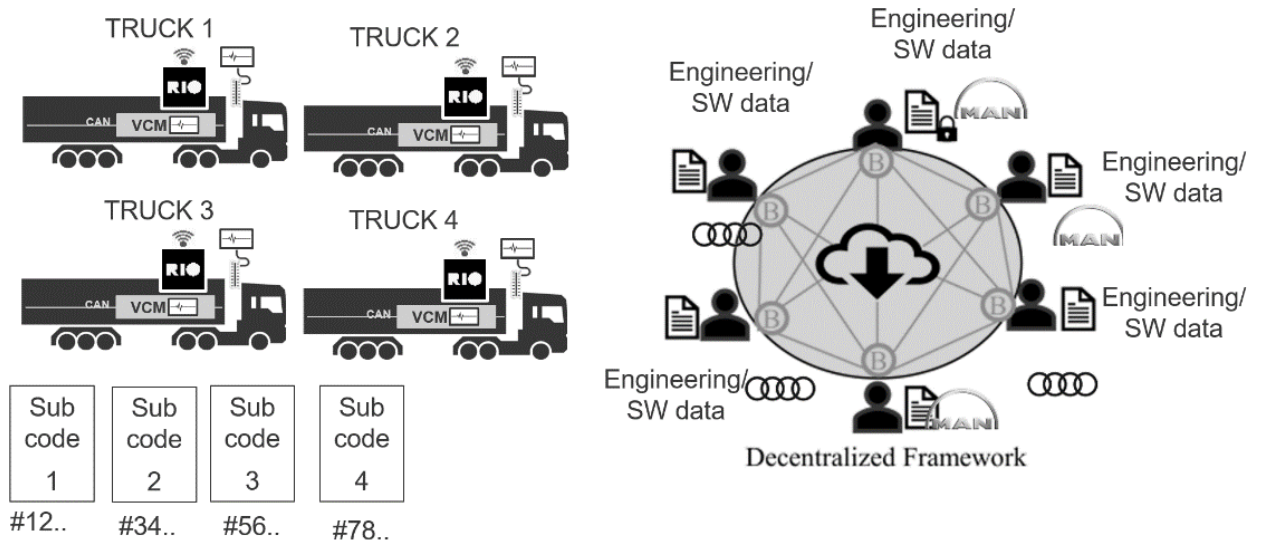

Figure 5. Data Chains for Decentralized Software data Management

\section{CONCLUSION}

This work proposes a holistic framework to ensure transparency, integrity and full traceability of software engineering data throughout the whole product lifecycle of automotive parts. The framework is based on the decentralized Blockchain technology in the area of MBSE with the associated product data and product life cycle management. A software Blockchain network is presented in order to ensure the traceability and integrity of the developed and deployed software in the connected products. This introduced structure is realized by sub- and main chains allowing for an optimized access and retrieval of the data. The concept development of the proposed approach is conducted in the frame of the "CarEngChainNet" research project based on real use cases from MAN Truck \& Bus. The defined use cases will serve in future as a platform to validate the developed framework and evaluate the vast benefits of Blockchain technology integration in the engineering process and identify other potential application fields. 


\section{REFERENCES}

Bogart, S. and Rice, K. (2015), The Blockchain Report: Welcome to the Internet of Value https://needham.bluematrix.com/sellside/EmailDocViewer?encrypt=4aaafaf1-d76e-4ee3-94067d0ad3c0d019\&mime=pdf\&co=needham\&id=sbogart@ @eedhamco.com\&source=mail\&utm_content=buff erOb432\&utm_medium=social\&utm_source=twitter.com\&utm_campaign=buffer (Accessed: 19 October 2020)

Glaser, F. and Bezzenberger, L. (2015), "Beyond Cryptocurrencies - A Taxonomy of Decentralized Consensus Systems”, ECIS 2015 Completed Research Papers. Paper 57 https://doi.org/10.18151/7217326

Heber, D. and Groll, M. (2017), "Towards A Digital Twin: How The Blockchain can foster E/E-Traceability in Consideration of Model-Based Systems Engineering", 21st International Conference on Engineering Design (ICED17), Vol. 3: Product, Services and Systems Design, Vancouver, Canada, 21.-25.08.2017.

Mattila, J. (2016), "The Blockchain Phenomenon - The Disruptive Potential of Distributed Consensus Architectures", ETLA Working Papers No 38. The Research Institute of the Finnish Economy http://pub.etla.fi/ETLA-Working-Papers-38.pdf (Accessed: 03 June 2016)

MHP and Riddle\&Code (2019) The Automotive Sector and Blockchain Available at: https://www.mhp.com/fileadmin/www.mhp.com/assets/pdf/MHP_Blockchain-in-Mobility_EN.pdf (Accessed: 23 May 2018)

MOBI (2019), MOBI Announces the first Vehicle Identity (VID) Standard on Blockchain [Press release]. 17.07.2019. Available at: https://dlt.mobi/wp-content/uploads/2020/04/Preview-MOBI-Vehicle-IdentityStandard-v1.0.pdf (Accessed: 19 October 2020)

Mullender, S. (1990), Introduction, in: Distributed Systems, Association for Computing Machinery Press, New York, USA. https://doi.org/10.1145/90417

Salehi, V. \& McMahon, C. (2009a), "Action Research into the use of parametric associative CAD systems in an industrial context”, International Conference on Engineering Design, ICED’09, Stanford University, Stanford, CA, USA United States, 24/08/09 - 27/08/09.

Salehi, V. \& McMahon, C. (2009b), "Development of a Generic Integrated Approach for Parametric Associative CAD Systems”, International Conference on Engineering Design, ICED’09, Stanford University, Stanford, CA, USA United States, 24/08/09 - 27/08/09.

Salehi, V. \& McMahon, C. (2009c), "Methodological Integration of Parametric Associative CAD Systems in Product Lifecycle Management (PLM) Environment”, ASME 2009 International Design Engineering Technical Conferences and Computers and Information in Engineering Conference, Volume 2: 29th Computers and Information in Engineering Conference, Parts A and B. San Diego, California, USA. August 30-September 2, 2009, pp. 505-514. https://doi.org/10.1115/DETC2009-86583

Salehi, V. \& McMahon, C. (2011a), "Development and application of an integrated approach for parametric associative CAD design in an industrial context", Computer-Aided Design and Applications, vol. 8, no. 2, pp. 225-236. https://doi.org/10.3722/cadaps.2011.225-236

Salehi, V. \& McMahon, C. (2011b), "Development of an evaluation framework for implementation of parametric associative methods in an industrial context", ICED 11 - 18th International Conference on Engineering Design - Impacting Society Through Engineering Design, Technical University of Denmark, Copenhagen, vol. 2, pp. 173-182, 15/08/11.

Salehi, V. \& McMahon, C. (2014), "Design of an Engine by means of a generic integrated approach of parametric associative CAD Systems and PLM", Computer Aided Design and application, Volume 2, Number 2, pp 220-231.

Salehi, V. and Wang, S. (2019), "Munich Agile MBSE Concept (MAGIC)", Design Society: International Conference on Engineering Design, Cambridge University Press, 1(1), pp. 3701-3710. http://doi.org/10.1017/dsi.2019.377

Salehi, V., Gross, F. and Taha, J. (2018), "Implementation of Systems Modelling Language (SysML) in Consideration of the consens Approach”, DESIGN 2018 15th International Design Conference, pp. 29872998. http://doi.org/10.21278/idc.2018.0146

Schoder, D. and Fischbach, K. (2002), Peer-to-Peer Wirtschaftsinformatik: Vol. 44, No. 6. Springer. (S. 587-589). http://doi.org/10.1007/BF03250877

Schollmeier, R. (2001), "A definition of peer-to-peer networking for the classification of peer-to-peer architectures and applications", First International Conference on Peer-to-Peer Computing, Linkoping, Sweden, pp. 101-102. http://doi.org/10.1109/P2P.2001.990434.

Swan, M. (2015), Blockchain: Blueprint for a New Economy (1st ed.), O’Reilly Media, Inc., Sebastopol, CA, USA

Walport, M. (2015), Distributed Ledger Technology: beyond block chain, 03.06.2016, UK Government Chief Scientific Adviser, London 